\title{
Chapter 1 \\ Note on the gonality of abstract modular curves
}

Anna Cadoret

\begin{abstract}
Let $S$ be a curve over an algebraically closed field $k$ of characteristic $p \geq 0$. To any family of representations $\rho=\left(\rho_{\ell}: \pi_{1}(S) \rightarrow \mathrm{GL}_{n}\left(\mathbb{F}_{\ell}\right)\right)$ indexed by primes $\ell \gg 0$ one can associate abstract modular curves $S_{\rho, 1}(\ell)$ and $S_{\rho}(\ell)$ which, in this setting, are the modular analogues of the classical modular curves $Y_{1}(\ell)$ and $Y(\ell)$. The main result of this paper is that, under some technical assumptions, the gonality of $S_{\rho}(\ell)$ goes to $+\infty$ with $\ell$. These technical assumptions are satisfied by $\mathbb{F}_{\ell}$-linear representations arising from the action of $\pi_{1}(S)$ on the étale cohomology groups with coefficients in $\mathbb{F}_{\ell}$ of the geometric generic fiber of a smooth proper scheme over $S$. From this, we deduce a new and purely algebraic proof of the fact that the gonality of $Y_{1}(\ell)$, for $p \nmid \ell\left(\ell^{2}-1\right)$, goes to $+\infty$ with $\ell$.
\end{abstract}

Key words: 2010 MSC Primary: 14H30, 14K99; Secondary: 14K10.

\subsection{Introduction}

Let $k$ be an algebraically closed field of characteristic $p \geq 0$ and $S$ a smooth, separated and connected curve over $k$ with generic point $\eta$. Let $\pi_{1}(S)$ denote its étale fundamental group. Fix an integer $n \geq 1$. For each prime $\ell \gg 0$, let $H_{\ell}$ be an $\mathbb{F}_{\ell}$ vector space of dimension $n$ on which $\pi_{1}(S)$ acts continuously. We will write $\rho$ for the family of the resulting $\mathbb{F}_{\ell}$-linear representations

$$
\rho_{\ell}: \pi_{1}(S) \rightarrow \mathrm{GL}\left(H_{\ell}\right) \simeq \mathrm{GL}_{n}\left(\mathbb{F}_{\ell}\right) \text {. }
$$

To such data, one can associate families of abstract modular curves $S_{\rho, 1}(\ell) \rightarrow S$ and $S_{\rho}(\ell) \rightarrow S$, see Section 1.2, which, in this setting, are the modular analogues

Anna Cadoret

Université Bordeaux 1, 351 Cours de la Libération, F 33405 TALENCE Cedex FRANCE e-mail: anna.cadoret@math.u-bordeaux1.fr 
of the classical modular curves $Y_{1}(\ell) \rightarrow Y(0)$ and $Y(\ell) \rightarrow Y(0)$ classifying $\ell$-torsion points and full level- $\ell$ structures of elliptic curves respectively.

The main examples of such representations we have in mind are the $\mathbb{F}_{\ell}$-linear representations arising from the action of $\pi_{1}(S)$ on the étale cohomology groups with coefficients in $\mathbb{F}_{\ell}$ of the geometric generic fiber of a smooth proper scheme over $S$. In particular, this includes those representations arising from the action of $\pi_{1}(S)$ on the group of $\ell$-torsion points of the geometric generic fiber of an abelian scheme over $S$, see Subsection 1.2.3.

The properties satisfied by these representations motivated, in [CT10b], the introduction of technical conditions on $\rho$, denoted by (A), (WA) and (AWA) for abelianization, weak abelianization and alternating weak abelianization respectively, (I) for isotriviality, (T) for tame and (U) for unipotent. See Subsection 1.2.2 for a precise formulation of these conditions.

Let $g_{\rho, 1}(\ell)$ and $g_{\rho}(\ell)$ (resp. $\gamma_{\rho, 1}(\ell)$ and $\gamma_{\rho}(\ell)$ ) denote the genus (resp. the $k$ gonality) of the abstract modular curves $S_{\rho, 1}(\ell)$ and $S_{\rho}(\ell)$ respectively. The main result of [CT10b] ([CT10b, Thm. 2.1]) asserts that, if conditions (AWA), (I), (U) are satisfied then:

$$
\lim _{\ell \rightarrow+\infty} g_{\rho, 1}(\ell)=+\infty \text {. }
$$

An intermediate step in the proof of this result is that, if conditions (WA), (I), (T) are satisfied then:

$$
\lim _{\ell \rightarrow+\infty} g_{\rho}(\ell)=+\infty \text {. }
$$

In this note, we prove that the same holds with gonality replacing genus, that is:

Theorem 1. If conditions (WA), (I), (T) are satisfied then:

$$
\lim _{\ell \rightarrow+\infty} \gamma_{\rho}(\ell)=+\infty
$$

The proof of Theorem 1 is purely algebraic and based on the equivariantprimitive decompositions introduced by A. Tamagawa in [T04] to estimate the gonality of Galois covers. The method, however, fails to prove:

Conjecture 2. Assume that conditions (WA), (T), (U) are satisfied. Then:

$$
\lim _{\ell \rightarrow+\infty} \gamma_{\rho, 1}(\ell)=+\infty
$$

Our method shows Conjecture 2 only when we restrict to $n=2$ and primes $\ell$ with $p \nmid \ell\left(\ell^{2}-1\right)$, or, more generally, for the variant of $S_{\rho, 1}(\ell)$ classifying points $v \in H_{\ell}$ whose $\pi_{1}(S)$-orbit generates a subspace of rank 2, see Proposition 14. This provides in particular an algebraic proof of the well-known fact, cf. [A96], [P07], that:

\section{Corollary 3.}

$$
\lim _{\substack{\ell \rightarrow+\infty \\ p \ell\left(\ell^{2}-1\right)}} \gamma_{Y_{1}(\ell)}=+\infty
$$


When $p=0$, it seems that variants of Theorem 1 can be proved by the techniques from differential geometry and Cayley-Schreier graph theory generalizing [A96] and developed in [EHK10].

Apart from their intrinsic geometric interest, statements as Theorem 1 and Conjecture 2 also have arithmetic consequences. In characteristic 0 , this follows from the following corollary of [F91].

Corollary 4. [Fr94]) Let $k$ be a finitely generated field of characteristic 0 and let $S$ be a smooth, proper, geometrically connected curve over $k$ with $k$-gonality $\gamma$. Then, for any integer $1 \leq d \leq\left[\frac{\gamma-1}{2}\right]$, the set of all closed points $s$ of $S$ with residue field $k(s)$ of degree $[k(s): k] \leq d$ is finite.

So, for instance, Conjecture 2 for $p=0$ combined with [CT10a, Prop. 3.18], to rule out the $\bar{k}$-isotrivial torsion points of $A_{\bar{\eta}}$, would imply:

For any finitely generated field $k$ of characteristic 0 , smooth, separated and geometrically connected curve $S$ over $k$, abelian scheme $A \rightarrow S$ and integer $d \geq 1$ the set of closed points $s$ of $S$ with degree $[k(s): k] \leq d$ and such that $A_{s}$ carries a $k(s)$-rational torsion point of order $\ell$ is finite for $\ell \gg 0$.

\section{Acknowledgements}

I am very indebted to Jakob Stix for his impressive editorial work (from which the exposition of this paper gained a lot) and for pointing out mathematical gaps in the last part of the proof of theorem 1 and in the proof of corollary 3. I am also grateful to Akio Tamagawa for his careful reading of the first version of this text as well as to the referee for his detailed and constructive report.

\subsection{Abstract modular curves}

We fix once and for all an algebraically closed field $k$ of characteristic $p \geq 0$. By a curve over $k$ we mean a connected, smooth and separated $k$-scheme of dimension 1 .

\subsubsection{Notation}

Let $S$ be a curve over $k$ with a geometric generic point $\bar{\eta}$ above its generic point $\eta \in S$. We will write $S \hookrightarrow S^{c p t}$ for the smooth compactification of $S$ and $\pi_{1}(S)$ for its étale fundamental group with base point $\bar{\eta}$. Fix an integer $n \geq 1$, and, for each prime $\ell \gg 0$, let $H_{\ell}$ be an $\mathbb{F}_{\ell}$-module of rank $n$ on which $\pi_{1}(S)$ acts. We will write $\rho$ for the family of the resulting $\mathbb{F}_{\ell}$-linear representations

$$
\rho_{\ell}: \pi_{1}(S) \rightarrow \mathrm{GL}\left(H_{\ell}\right) \simeq \mathrm{GL}_{n}\left(\mathbb{F}_{\ell}\right) .
$$


For every prime $\ell \gg 0$, set $G_{\ell}=\operatorname{im}\left(\rho_{\ell}\right)$ and for any subgroup $U \subset G_{\ell}$, the abstract modular curve associated to $U$ is the connected étale cover $S_{U} \rightarrow S$ corresponding to the open subgroup $\rho_{\ell}^{-1}(U) \subset \pi_{1}(S)$. We write $g_{S_{U}}$ and $\gamma_{S_{U}}$ for genus and gonality of $S_{U}$ respectively.

Remark 5. As we are only interested in the asymptotic behaviour of abstract modular curves, it is enough to consider only big enough primes $\ell$. Furthermore, in practice, $H_{\ell}$ will be an étale cohomology group $H^{i}\left(X_{\bar{\eta}}, \mathbb{F}_{\ell}\right)$ for some smooth proper morphism $X \rightarrow S$ with connected geometric generic fibre $X_{\bar{\eta}}$. In particular, the dimension of $H^{i}\left(X_{\bar{\eta}}, \mathbb{F}_{\ell}\right)$ may become constant only for $\ell \gg 0$, see Subsection 1.2.2.

In the following, we will consider only specific classes of abstract modular curves of two kinds. First, for $v \in H_{\ell}$ we denote by $S_{v} \rightarrow S$ the abstract modular curve associated to the stabilizer of $G_{\ell, v} \subset G_{\ell}$ of $v$, and let $g_{v}$ and $\gamma_{v}$ denote its genus and gonality respectively.

Secondly, for a $\pi_{1}(S)$-submodule $M \subset H_{\ell}$, we denote by $S_{M} \rightarrow S$ the abstract modular curve associated to $\operatorname{Fix}(M):=\left\{g \in G_{\ell}|g|_{M}=\operatorname{Id}_{M}\right\}$, and let $g_{M}$ and $\gamma_{M}$ denote its genus and gonality respectively. The connected étale cover $S_{M} \rightarrow S$ is Galois with Galois group $G_{M}=G_{\ell} /$ Fix $(M)$, which is the image of the induced representation $\rho_{M}: \pi_{1}(S) \rightarrow \mathrm{GL}(M)$.

For $v \in H_{\ell}$ and the $\pi_{1}(S)$-submodule $M(v):=\mathbb{F}_{\ell}\left[G_{\ell} \cdot v\right] \subset H_{\ell}$ generated by $v$, the cover $S_{M(v)} \rightarrow S$ is the Galois closure of $S_{v} \rightarrow S$.

Let $\mathscr{F}=\left(\mathscr{F}_{\ell}\right)$ denote a sequence of non-empty families of subgroups of $G_{\ell}$. We will say that:

$$
S_{\rho, \mathscr{F}}(\ell):=\bigsqcup_{U \in \mathscr{F}_{\ell}} S_{U} \rightarrow S
$$

is the abstract modular curve associated with $\mathscr{F} \ell$ and define:

$$
\begin{aligned}
& d_{\rho, \mathscr{F}}(\ell):=\min \left\{\left[G_{\ell}: U\right] ; U \in \mathscr{F}_{\ell}\right\} \\
& g_{\rho, \mathscr{F}}(\ell):=\min \left\{g_{S_{U}} ; U \in \mathscr{F}_{\ell}\right\} \\
& \gamma_{\rho, \mathscr{F}}(\ell):=\min \left\{\gamma_{S_{U}} ; U \in \mathscr{F}_{\ell}\right\}
\end{aligned}
$$

which we call the degree, genus and gonality of the abstract modular curve $S_{\rho, \mathscr{F}}(\ell)$. Following the notation for the usual modular curves, we will write:

$$
S_{\rho, 1}(\ell), d_{\rho, 1}(\ell), g_{\rho, 1}(\ell), \gamma_{\rho, 1}(\ell)
$$

when $\mathscr{F}_{\ell}$ is the family of all stabilizers $G_{\ell, v}$ for $0 \neq v \in H_{\ell}$, and

$$
S_{\rho}(\ell), d_{\rho}(\ell), g_{\rho}(\ell), \gamma_{\rho}(\ell)
$$

when $\mathscr{F}_{\ell}$ is the family of all $\operatorname{Fix}(M)$, for $0 \neq M \subset H_{\ell}$. Note that by construction

$$
d_{\rho}(\ell) \geq d_{\rho, 1}(\ell), g_{\rho}(\ell) \geq g_{\rho, 1}(\ell) \text { and } \gamma_{\rho}(\ell) \geq \gamma_{\rho, 1}(\ell) \text {. }
$$




\subsubsection{Conditions (WA), (I), (T)}

Given an integer $1 \leq m \leq n$ and a $\pi_{1}(S)$-submodule $M \subset \Lambda^{m} H_{\ell}$, write again

$$
\rho_{M}: \pi_{1}(S) \rightarrow \mathrm{GL}(M)
$$

for the induced representation. We consider the following technical conditions on $\rho:$

(WA) For any open subgroup $\Pi \subset \pi_{1}(S)$, there exists an integer $B_{\Pi} \geq 1$ such that, for every prime $\ell$, integer $1 \leq m \leq n$ and $\Pi$-submodule $M \subset \Lambda^{m} H_{\ell}$, one has:

$$
\rho_{M}(\Pi) \text { abelian of prime-to- } \ell \text { order } \Rightarrow\left|\rho_{M}(\Pi)\right| \leq B_{\Pi} .
$$

(WA)' For any open subgroup $\Pi \subset \pi_{1}(S)$, there exists an integer $B_{\Pi} \geq 1$ such that, for every prime $\ell$, integer $1 \leq m \leq n$ and $\Pi$-submodule $M \subset \Lambda^{m} H_{\ell}$, one has:

$$
\rho_{M}(\Pi) \text { abelian } \Rightarrow\left|\rho_{M}(\Pi)\right| \leq B_{\Pi} .
$$

(I) For any open subgroup $\Pi \subset \pi_{1}(S)$ the $\mathbb{F}_{\ell}$-submodule $H_{\ell}^{\Pi}$ of fixed vectors under $\Pi$ is trivial for $\ell \gg 0$.

(T) For any $P \in S^{c p t} \backslash S$ there exists an open subgroup $T_{P}$ of the inertia group $I_{P} \subset \pi_{1}(S)$ at $P$ such that $\rho_{\ell}\left(T_{P}\right)$ is tame for $\ell \gg 0$.

In $[\mathrm{CT} 10 \mathrm{~b}]$, we introduce an additional condition (U), which asserts that for any $P \in S^{c p t} \backslash S$ there exists an open subgroup $U_{P}$ of the inertia group $I_{P} \subset \pi_{1}(S)$ at $P$ such that $\rho_{\ell}\left(U_{P}\right)$ is unipotent for $\ell \gg 0$. Condition (U) is stronger than condition (T); we will not use it in the following.

See [CT10b, §2.3] for more details, in particular for the following lemma.

Lemma 6. ([CT10b, Lem. 2.2, 2.3 and 2.4])

(1) Assume that condition (T) is satisfied. Set $K:=\bigcap_{\ell} \operatorname{ker}\left(\rho_{\ell}\right)$. Then $\pi_{1}(S) / K$ is topologically finitely generated.

(2) Conditions (I) and (T) imply $\lim _{\ell \rightarrow+\infty} d_{\rho, 1}(\ell)=+\infty$.

(3) Conditions (I), (T) and (WA) imply condition (WA)'.

Assume that conditions (I), (T) and (WA) are satisfied. Since $d_{\rho}(\ell) \geq d_{\rho, 1}(\ell)$, it follows from Lemma 6 (2) and (3) that for $\ell \gg 0$ and any $\pi_{1}(S)$-submodule $0 \neq M \subset$ $H_{\ell}$ the group $G_{M}$ cannot be abelian.

Corollary 7. Assume that conditions (I), (T) and (WA) hold. Then, for any integer $B \geq 1$, for every $\pi_{1}(S)$-submodule $0 \neq M \subset H_{\ell}$ and for every abelian subgroup $A$ of $G_{M}$ one has $\left[G_{M}: A\right] \geq B$ for $\ell \gg 0$.

Proof. Otherwise, there exists an integer $B \geq 1$ and an infinite set of primes $\mathscr{S}$ such that, for every $\ell \in \mathscr{S}$, there exists a $\pi_{1}(S)$-submodule $0 \neq M_{\ell} \subset H_{\ell}$ and an abelian subgroup $A_{\ell}$ of $G_{M_{\ell}}$ with $\left[G_{M_{\ell}}: A_{\ell}\right] \leq B$. But, since it follows from Lemma 6 (1) 
that $\pi_{1}(S)$ acts through a topologically finitely generated quotient, there are only finitely many isomorphism classes of connected étale covers of $S$ corresponding to the $\rho_{M_{\ell}}^{-1}\left(A_{\ell}\right) \subset \pi_{1}(S), \ell \in \mathscr{S}$. Hence at least one of them, say $S^{\prime} \rightarrow S$, appears infinitely many times. Up to base-changing by $S^{\prime} \rightarrow S$, we may assume that $G_{M_{\ell}}$ is abelian for infinitely many $\ell \in \mathscr{S}$, which contradicts Lemma 6 (2) and (3).

\subsubsection{Etale cohomology}

Let $X \rightarrow S$ be a smooth, proper morphism with geometrically connected fibers. For every integer $i \geq 0$ the $\mathbb{F}_{\ell}$-rank $n_{i, \ell}$ of $H_{\ell}^{i}:=\mathrm{H}^{i}\left(X_{\bar{\eta}}, \mathbb{F}_{\ell}\right)$ is finite and independent of $\ell$ for $\ell \gg 0$. Indeed, when $p=0$, this follows from the comparison isomorphism between Betti and étale cohomology with finite coefficients and the fact that Betti cohomology with coefficient in $\mathbb{Z}$ is finitely generated. More generally, when $p \geq 0$, this follows from the fact that $\ell$-adic cohomology with coefficients in $\mathbb{Z}_{\ell}$ is torsion free for $\ell \gg 0$ [G83] and that the $\mathbb{Q}_{\ell}$-rank of $\ell$-adic cohomology with coefficients in $\mathbb{Q}_{\ell}$ is independent of $\ell$. So, we will simply write $n_{i}$ instead of $n_{i, \ell}$ for $\ell \gg 0$.

For each $i \geq 1$ and $\ell \gg 0$, the action of $\pi_{1}(S)$ on $H_{\ell}^{i}$ gives rise to a family $\rho^{i}=\left(\rho_{\ell}^{i}\right)$ of $n_{i}$-dimensional $\mathbb{F}_{\ell}$-linear representations

$$
\rho_{\ell}^{i}: \pi_{1}(S) \rightarrow \mathrm{GL}\left(H_{\ell}^{i}\right) \simeq \mathrm{GL}_{n_{i}}\left(\mathbb{F}_{\ell}\right) .
$$

It follows from [CT10b, Thm. 2.4] that the families $\rho^{i}$ for $i \geq 1$ satisfy conditions (T) and (WA). As for condition (I), if $X_{\eta}$ is projective over $k(\eta)$ then, for $i=1$ it can be ensured by the condition:

$$
\mathrm{Pic}_{X_{\bar{\eta}} / k(\bar{\eta})}^{0} \text { contains no non-trivial } k \text {-isotrivial abelian subvarieties. }
$$

\subsection{Technical preliminaries}

The proof of Theorem 1 is based on a combination of Lemma 6 with the use of E-P decomposition and group-theoretic ingredients. We gather the results we will need in Subsections 1.3.1, 1.3.2 and 1.3.3 respectively.

\subsubsection{E-P decompositions}

Consider a diagram of proper curves over $k$ 


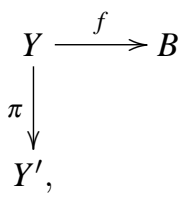

where $f: Y \rightarrow B$ is a non-constant morphism of proper curves over $k$ and $\pi: Y \rightarrow Y^{\prime}$ is a G-cover with group $G$ (that is $G$ acts faithfully on $Y$ and $\pi: Y \rightarrow Y^{\prime}$ is the quotient morphism $Y \rightarrow Y / G)$. We will say that a pair of maps $(\pi, f)$ as in (1.1) is equivariant if for any $\sigma \in G$ there exists $\sigma_{B} \in \operatorname{Aut}_{k}(B)$ such that $f \circ \sigma=\sigma_{B} \circ f$ and that $(\pi, f)$ as in (1.1) is primitive if it does not have any equivariant nontrivial subdiagram that is, more precisely, if for any commutative diagram (1.2) of morphisms of proper curves over $k$

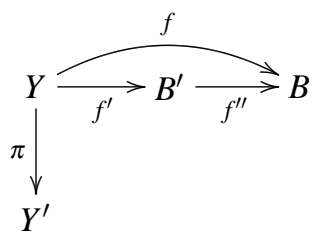

with $f^{\prime}$ and $f^{\prime \prime}$ of degree $\geq 2$, the pair $\left(\pi, f^{\prime}\right)$ is not equivariant.

We will resort to the following corollary of the Castelnuovo-Severi inequality.

Lemma 8. ([T04, Thm. 2.4]). If the pair of maps $(\pi, f)$ as in $(1.1)$ is primitive then:

$$
\operatorname{deg}(f) \geq \sqrt{\frac{g_{Y}+1}{g_{B}+1}} .
$$

For a pair $(\pi, f)$ as in diagram (1.1), among all equivariant decompositions, i.e., diagrams as (1.2) with the pair $\left(\pi, f^{\prime}\right)$ equivariant, we choose a pair $\left(\pi, f^{\prime}: Y \rightarrow C\right)$ with $\operatorname{deg}\left(f^{\prime}\right)$ maximal. This exists as $(\pi, \mathrm{id})$ is equivariant and $\operatorname{deg}\left(f^{\prime}\right) \leq \operatorname{deg}(f)$ is bounded. By definition, the action of $G$ on $Y$ induces an action on $C$, hence we obtain a homomorphism $G \rightarrow \operatorname{Aut}_{k}(C)$. We set $\bar{G}=G / K$ where

$$
K:=\operatorname{Ker}\left(G \rightarrow \operatorname{Aut}_{k}(C)\right) .
$$

Then diagram (1.1) for $(\pi, f)$ can be enriched to a commutative diagram with respect to the maximal equivariant decomposition $\left(\pi, f^{\prime}\right)$ as follows:

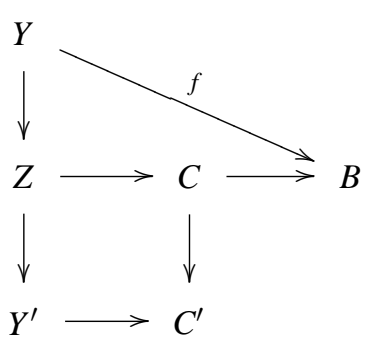


where the vertical maps $Y \rightarrow Z=Y / K, Z \rightarrow Y^{\prime}=Z / \bar{G}$ and $C \rightarrow C^{\prime}=C / \bar{G}$ are the quotient morphisms. By construction, the pair $\left(Z \rightarrow Y^{\prime}, Z \rightarrow C\right)$ is equivariant and the pair $\left(C \rightarrow C^{\prime}, C \rightarrow B\right)$ is primitive. We will call such a decomposition an equivariant-primitive decomposition (E-P decomposition for short).

\subsubsection{Review of the classification of finite subgroups of $\mathrm{SL}_{2}$}

We remind that $k$ is a fixed algebraically closed field of characteristic $p \geq 0$. Then we have the following description of finite subgroups of $\mathrm{SL}_{2}(k)$.

Theorem 9. ([Su82, Thm. 3.6.17]) A finite subgroup $G$ of $\mathrm{SL}_{2}(k)$ is one from the following list:

(1) a cyclic group,

(2) for some $n \geq 2$ a group with presentation

$$
\left\langle x, y \mid x^{n}=y^{2}, y^{-1} x y=x^{-1}\right\rangle
$$

(3) $\mathrm{SL}_{2}(3)$, or $\mathrm{SL}_{2}(5)$,

(4) the representation group $\hat{\mathscr{S}}_{4}$ of the permutation group $\mathscr{S}_{4}$ in which transpositions lift to elements of order 4 ,

(5) an extension

$$
1 \rightarrow A \rightarrow G \rightarrow Q \rightarrow 1
$$

where $A$ is an elementary abelian p-group and $Q$ is a cyclic group of prime-to- $p$ order,

(6) a dihedral group,

(7) $\mathrm{SL}_{2}\left(k_{r}\right)$, where $k_{r}$ denotes the subfield of $k$ with $p^{r}$ elements,

(8) $\left\langle\mathrm{SL}_{2}\left(k_{r}\right), d_{\pi}\right\rangle$, where $d_{\pi}$ is the scalar matrix with diagonal entries given by a $\pi \in k$ such that $k_{r}(\pi)$ has $p^{2 r}$ elements and $\pi^{2}$ is a generator of $k_{r}^{\times}$.

Case (6) occurs only when $p=2$ and cases (7) and (8) occur only when $p>0$.

We will use two easy corollaries of Theorem 9. Namely, observing that when $k$ is algebraically closed $\mathrm{PGL}_{2}(k)=\mathrm{PSL}_{2}(k)$, we get the well known corollary:

Corollary 10. A finite subgroup $G$ of $\mathrm{PGL}_{2}(k)$ is of the following form:

(1) a cyclic group,

(2) a dihedral group,

(3) $\mathscr{A}_{4}, \mathscr{S}_{4}, \mathscr{A}_{5}$,

(4) an extension

$$
1 \rightarrow A \rightarrow G \rightarrow Q \rightarrow 1
$$

where $A$ is an elementary abelian p-group and $Q$ is a cyclic group of prime-to- $p$ order,

(5) $\operatorname{PSL}_{2}\left(k_{r}\right)$, 
(6) $\mathrm{PGL}_{2}\left(k_{r}\right)$.

The last three cases occur only when $p>0$.

Also, regarding $\mathrm{SL}_{2}\left(\mathbb{F}_{\ell}\right)$ as a subgroup of $\mathrm{SL}_{2}\left(\overline{\mathbb{F}}_{\ell}\right)$ and ruling out the groups that cannot lie in $\mathrm{SL}_{2}\left(\mathbb{F}_{\ell}\right)$, we get:

Corollary 11. Assume that $\ell \geq 5$. A subgroup of $\mathrm{SL}_{2}\left(\mathbb{F}_{\ell}\right)$ is isomorphic to one of the following.

(1) a cyclic group,

(2) for some $n \geq 2$ a group with presentation

$$
\left\langle x, y \mid x^{n}=y^{2}, y^{-1} x y=x^{-1}\right\rangle,
$$

(3) $\mathrm{SL}_{2}\left(\mathbb{F}_{3}\right)$, or $\mathrm{SL}_{2}\left(\mathbb{F}_{5}\right)$,

(4) the representation group $\hat{\mathscr{S}}_{4}$ of the permutation group $\mathscr{S}_{4}$ in which transpositions lift to elements of order 4 ,

(5) a semi-direct product $\mathbb{F}_{\ell} \rtimes C$ contained in a Borel subgroup with $C$ a cyclic group of prime-to- $\ell$ order,

(6) $\mathrm{SL}_{2}\left(\mathbb{F}_{\ell}\right)$.

\subsubsection{A group-theoretic lemma}

The following lemma provides a practical condition for a finite group to contain a large normal abelian subgroup.

Lemma 12. Let $G$ be a finite group and assume that $G$ fits into a short exact sequence of finite groups

$$
1 \rightarrow N \rightarrow G \rightarrow Q \rightarrow 1
$$

with $Q$ abelian and generated by $\leq r$ elements. Then the group $G$ contains a normal abelian subgroup A with index

$$
[G: A] \leq \mu(Z(N))^{r} \cdot|\operatorname{Aut}(N)|,
$$

where $\mu(Z(N))$ denotes the least common multiple of the order of the elements in the center $Z(N)$ of $N$.

Proof. The short exact sequence $(*)$ induces by conjugation representations

$$
\tilde{\phi}: G \rightarrow \operatorname{Aut}(N) \quad \text { and } \quad \phi: Q \rightarrow \operatorname{Out}(N)
$$

and induces on the centralizer $Z_{G}(N)=\operatorname{ker}(\tilde{\phi})$ of $N$ in $G$ the structure of a central extension

$$
1 \rightarrow Z(N) \rightarrow Z_{G}(N) \rightarrow \operatorname{ker}(\phi) \rightarrow 1 .
$$


Because the extension is central, taking the commutator of lifts to $Z_{G}(N)$ defines an alternating bilinear form $[$,$] on \operatorname{ker}(\phi)$ with values in $Z(N)$. The radical of $[$,

$$
R=\left\{q \in \operatorname{ker}(\phi) ;\left[q, q^{\prime}\right]=0 \text { for all } q^{\prime} \in \operatorname{ker}(\phi)\right\} \subset \operatorname{ker}(\phi),
$$

contains $\mu(Z(N)) \operatorname{ker}(\phi)$. We find an extension

$$
1 \rightarrow Z(N) \rightarrow Z\left(Z_{G}(N)\right) \rightarrow R \rightarrow 1
$$

where $A=Z\left(Z_{G}(N)\right)$ is the center of $Z_{G}(N)$. Since $N$ is normal in $G$, the abelian group $A$ is also normal in $G$. We can estimate the index $[G: A]$ as

$$
\begin{gathered}
\quad[G: A]=\frac{|G|}{\left|Z_{G}(N)\right|} \cdot \frac{\left|Z_{G}(N)\right|}{|A|} \leq|\operatorname{Aut}(N)| \cdot \frac{|\operatorname{ker}(\phi)|}{|R|} \\
\leq|\operatorname{Aut}(N)| \cdot \frac{|\operatorname{ker}(\phi)|}{|\mu(Z(N)) \operatorname{ker}(\phi)|} \leq|\operatorname{Aut}(N)| \cdot \mu(Z(N))^{r}
\end{gathered}
$$

since $\operatorname{ker}(\phi) \subset Q$ is also generated by $\leq r$ elements.

\subsection{Proof of Theorem 1}

Observe first that if $S^{\prime} \rightarrow S$ is any connected finite étale cover then $\pi_{1}\left(S_{M}^{\prime}\right)=$ $\pi_{1}\left(S_{M}\right) \cap \pi_{1}\left(S^{\prime}\right)$. In particular, one has:

$$
\gamma_{S_{M}} \leq \gamma_{S_{M}^{\prime}} \leq \gamma_{S_{M}} \operatorname{deg}\left(S_{M}^{\prime} \rightarrow S_{M}\right) \leq \gamma_{S_{M}} \operatorname{deg}\left(S^{\prime} \rightarrow S\right)
$$

and, as a result, $\lim _{\ell \rightarrow+\infty} \gamma_{\left.\rho\right|_{\pi_{1}\left(S^{\prime}\right)}}(\ell)=+\infty$ if and only if $\lim _{\ell \rightarrow+\infty} \gamma_{\rho}(\ell)=+\infty$. This allows to perform arbitrary base changes by connected étale covers. In particular, from condition (T), one may assume that $\pi_{1}(S)$ acts through its tame quotient $\pi_{1}^{t}(S)$.

For every prime $\ell$, consider a $\pi_{1}(S)$-submodule $0 \neq M_{\ell} \subset H_{\ell}$ such that $\gamma_{M_{\ell}}=$ $\gamma_{\rho}(\ell)$. We thus have a diagram of proper curves over $k$

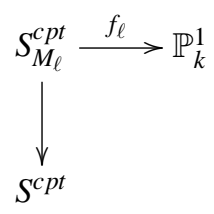

with $\operatorname{deg}\left(f_{\ell}\right)=\gamma_{\rho}(\ell)$. We can consider an E-P decomposition of (1.4) 


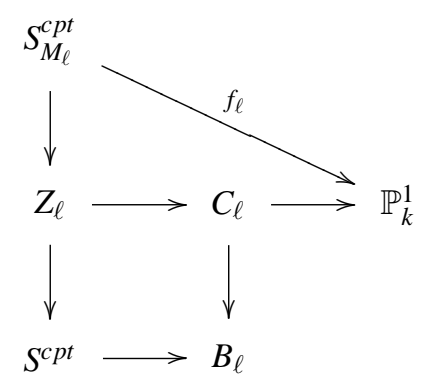

where $S_{M_{\ell}}^{c p t} \rightarrow Z_{\ell}=S_{M_{\ell}}^{c p t} / K_{\ell}$ and, with $\bar{G}_{M_{\ell}}=G_{M_{\ell}} / K_{\ell}$ faithfully acting on $C_{\ell}$, also $C_{\ell} \rightarrow B_{\ell}=C_{\ell} / \bar{G}_{M_{\ell}}$ are the respective quotient maps.

If $\gamma_{\rho}(\ell)$ does not diverge, then there exists an infinite subset $\mathscr{S}$ of primes and an integer $\gamma \geq 1$ such that $\gamma_{\rho}(\ell) \leq \gamma$ for all $\ell \in \mathscr{S}$. In particular $\left|K_{\ell}\right| \leq \gamma$, hence

$$
\left|\bar{G}_{M_{\ell}}\right| \geq \frac{d_{\rho}(\ell)}{\left|K_{\ell}\right|} \geq \frac{d_{\rho}(\ell)}{\gamma} .
$$

So, from Lemma 6 (2) one has $\lim _{\substack{\ell \rightarrow+\infty \\ \ell \in \mathscr{S}}}\left|\bar{G}_{M_{\ell}}\right|=+\infty$.

To get the contradiction, we distinguish between three cases. In the first case we assume that $g_{C_{\ell}} \geq 2$ for all but finitely many $\ell \in \mathscr{S}$. Since by [St73] the size of the automorphism group of a genus $g \geq 2$ curve over an algebraically closed field of characteristic $p$ is bounded by $P_{p}(g)$ for a polynomial $P_{p}(T) \in \mathbb{Z}[T]$ depending only on $p$, we find for $\ell \in \mathscr{S}$ that $\left|\bar{G}_{M_{\ell}}\right| \leq P_{p}\left(g_{C_{\ell}}\right)$, which forces

$$
\lim _{\substack{\ell \rightarrow+\infty \\ \ell \in \mathscr{S}}} g_{C_{\ell}}=+\infty
$$

But from Lemma 8 applied to the primitive pair $\left(C_{\ell} \rightarrow B_{\ell}, C_{\ell} \rightarrow \mathbb{P}_{k}^{1}\right)$ in diagram (1.4), one has

$$
\gamma_{\rho}(\ell)=\operatorname{deg}\left(f_{\ell}\right) \geq \operatorname{deg}\left(C_{\ell} \rightarrow \mathbb{P}_{k}^{1}\right) \geq \sqrt{g_{C_{\ell}}+1}
$$

which therefore also diverges for $\ell \in \mathscr{S}$ contradicting the choice of $\mathscr{S}$.

If we are not in the first case, then $g_{C_{\ell}} \leq 1$ for infinitely many $\ell \in \mathscr{S}$. In the second case, we assume that for infinitely many $\ell \in \mathscr{S}$, and in fact by replacing $\mathscr{S}$ by a subset, that for all $\ell \in \mathscr{S}$ we have $g_{C_{\ell}}=1$. Then for $\ell \in \mathscr{S}$, the group $\bar{G}_{M_{\ell}}$ is an extension

$$
1 \rightarrow A_{\ell} \rightarrow \bar{G}_{M_{\ell}} \rightarrow Q_{\ell} \rightarrow 1
$$

with $A_{\ell}$ a finite quotient of $\hat{\mathbb{Z}}^{2}$ and $\left|Q_{\ell}\right| \leq 24$. Since by Lemma $6 \pi_{1}(S)$ acts through a topologically finitely generated quotient, there are only finitely many isomorphism classes of étale covers of $S$ with degree $\leq 24$ corresponding to the inverse image of $A_{\ell}$ via 


$$
\pi_{1}(S) \stackrel{\rho_{M_{\ell}}}{\rightarrow} G_{M_{\ell}} \rightarrow \bar{G}_{M_{\ell}}
$$

So, by replacing $S$ by the composite of all these étale covers of degree $\leq 24$, we may assume that $\bar{G}_{M_{\ell}}=A_{\ell}$ for all $\ell \in \mathscr{S}$. Now Lemma 12 applied to

$$
1 \rightarrow K_{\ell} \rightarrow G_{M_{\ell}} \rightarrow A_{\ell} \rightarrow 1
$$

shows, since $\left|K_{\ell}\right| \leq \gamma$, that $G_{M_{\ell}}$ has an abelian subgroup of index bounded above independently of $\ell \in \mathscr{S}$ in contradiction to Corollary 7 .

In the last case we can and do assume that $g_{C_{\ell}}=0$ for all $\ell \in \mathscr{S}$. As above, Corollary 7 shows that the subgroup $\bar{G}_{M_{\ell}} \subset \operatorname{Aut}\left(C_{\ell}\right) \cong \mathrm{PGL}_{2}(k)$ can be only of type (4), (5) or (6) as in Corollary 10 for $\ell \gg 0$, and $\ell \in \mathscr{S}$. This occurs only if $p>0$. Without loss of generality, by replacing $\mathscr{S}$ by an infinite subset, we may assume that $\bar{G}_{M_{\ell}}$ is of the same type for all $\ell \in \mathscr{S}$. To rule out these cases, we are going to use the following theorem.

Theorem 13 ([N87, Thm. C]). For any integer $n \geq 1$ there exists an integer $d(n) \geq 1$ such that for any prime $\ell \geq n$, integer $m \leq n$ and subgroup $G$ of $\mathrm{GL}_{m}\left(\mathbb{F}_{\ell}\right)$ the following holds. Let $G^{+}$denote the (normal) subgroup of $G$ generated by the elements of order $\ell$ in $G$. Then, there exists an abelian subgroup $A \subset G$ such that $A G^{+}$is normal in $G$ and $\left[G: A G^{+}\right] \leq d(n)$.

Assume that $\bar{G}_{M_{\ell}}$ is of type (4) for all $\ell \in \mathscr{S}$, that is of the form

$$
(\mathbb{Z} / p)^{r_{\ell}} \rtimes Z / N_{\ell}
$$

for some integers $r_{\ell}, N_{\ell} \geq 1$ with $p \nmid N_{\ell}$.

Claim. There exists an integer $r(n) \geq 1$ such that $r_{\ell} \leq r(n)$ for $\ell \gg 0$ in $\mathscr{S}$.

Proof. Let $T_{\ell}$ denote the inverse image of $(\mathbb{Z} / p)^{r_{\ell}}$ in $G_{M_{\ell}}$ that is $T_{\ell}$ fits into the short exact sequence of finite groups

$$
1 \rightarrow K_{\ell} \rightarrow T_{\ell} \rightarrow(\mathbb{Z} / p)^{r_{\ell}} \rightarrow 1 .
$$

Because $\left|K_{\ell}\right| \leq \gamma$ we see that $\ell$ does not divide $\left|T_{\ell}\right|$ for $\ell \gg 0$ and, in particular, that $T_{\ell}^{+}$is trivial. Theorem 13 implies that $T_{\ell}$ fits into a short exact sequence

$$
1 \rightarrow A_{\ell} \rightarrow T_{\ell} \rightarrow Q_{\ell} \rightarrow 1
$$

with $A_{\ell}$ abelian and $\left|Q_{\ell}\right| \leq d(n)$. In turn, $A_{\ell}$ fits into the sort exact sequence

$$
1 \rightarrow K_{\ell} \cap A_{\ell} \rightarrow A_{\ell} \rightarrow(\mathbb{Z} / p)^{s_{\ell}} \rightarrow 1
$$

with $s_{\ell} \leq r_{\ell}$. In particular, $A_{\ell}$ is an abelian subgroup of $\mathrm{GL}\left(M_{\ell}\right)$ of prime-to- $\ell$ order and of $\mathbb{Z}$-rank $\geq s_{\ell}$. This implies $s_{\ell} \leq n$ since any abelian subgroup $A$ of order primeto- $\ell$ in $\mathrm{GL}_{n}\left(\mathbb{F}_{\ell}\right)$ is conjugate in $\mathrm{GL}_{n}\left(\overline{\mathbb{F}}_{\ell}\right)$ to a diagonal torus. So the claim follows from $r_{\ell} \leq s_{\ell}+\log _{p}\left|Q_{\ell}\right|$ and the bounds for $s_{\ell}$ and $\left|Q_{\ell}\right| \leq d(n)$. 
By the claim and Lemma 12 , the group $\bar{G}_{M_{\ell}}$ contains a normal abelian subgroup $A_{\ell}$ with index bounded by

$$
\left[\bar{G}_{M_{\ell}}: A_{\ell}\right] \leq p \cdot\left|\mathrm{GL}_{r(n)}\left(\mathbb{F}_{p}\right)\right| .
$$

Invoking again that $\pi_{1}(S)$ acts through a topologically finitely generated quotient, without loss of generality we may assume that $\bar{G}_{M_{\ell}}=A_{\ell}$ and then, as above the contradiction follows from the bound $\left|K_{\ell}\right| \leq \gamma$, Lemma 12 and Corollary 7 .

Assume now that $\bar{G}_{M_{\ell}}$ is of type (5) or (6) for all $\ell \in \mathscr{S}$, that is either $\operatorname{PSL}_{2}\left(k_{r_{\ell}}\right)$ or $\mathrm{PGL}_{2}\left(k_{r_{\ell}}\right)$ for some integer $r_{\ell} \geq 1$.

For any non zero vector $v \in M_{\ell}$ the cover $S_{M(v)} \rightarrow S$ is a quotient of $S_{M_{\ell}} \rightarrow S$ hence $\gamma_{S_{M(v)}} \leq \gamma_{S_{\ell}}$. So, without loss of generality, we may assume that $M_{\ell}$ is a simple $\pi_{1}(S)$-module. In particular, there exists a non zero vector $v \in M_{\ell}$ such that $M_{\ell}=M(v)$ and $M_{\ell}^{+}:=\mathbb{F}_{\ell}\left[G_{M_{\ell}}^{+} v\right] \subset M$ is a simple $G_{M_{\ell}}^{+}$-submodule.

Claim. The group $G_{M_{\ell}}^{+}$is nontrivial for $\ell \gg 0, \ell \in \mathscr{S}$.

Proof. Theorem 13 applied to $G_{M_{\ell}} \subset \mathrm{GL}\left(M_{\ell}\right)$ shows that one can write $G_{M_{\ell}} / G_{M_{\ell}}^{+}$ as an extension

$$
1 \rightarrow A_{\ell} G_{M_{\ell}}^{+} / G_{M_{\ell}}^{+} \rightarrow G_{M_{\ell}} / G_{M_{\ell}}^{+} \rightarrow Q_{\ell} \rightarrow 1
$$

with $A_{\ell} G_{M_{\ell}}^{+} / G_{M_{\ell}}^{+}$abelian and $\left|Q_{\ell}\right| \leq d(n)$, because $\operatorname{dim}_{\mathbb{F}_{\ell}}\left(M_{\ell}\right) \leq n$. As a result, if $G_{M_{\ell}}^{+}=1$, we get a contradiction to Corollary 7. This proves the claim.

Since $\mathrm{PSL}_{2}\left(k_{r_{\ell}}\right)$ is simple and the only nontrivial normal subgroups of $\mathrm{PGL}_{2}\left(k_{r_{\ell}}\right)$ are $\operatorname{PSL}_{2}\left(k_{r_{\ell}}\right)$ and $\mathrm{PGL}_{2}\left(k_{r_{\ell}}\right)$, the second claim implies that the normal subgroup

$$
\bar{G}_{M_{\ell}}^{+}:=G_{M_{\ell}}^{+} / G_{M_{\ell}}^{+} \cap K_{\ell}
$$

of $\bar{G}_{M_{\ell}}$ contains $\operatorname{PSL}_{2}\left(k_{r_{\ell}}\right)$.

Claim. $Z_{\ell}:=K_{\ell} \cap G_{M_{\ell}}^{+}$is a central subgroup of $G_{M_{\ell}}^{+}$for $\ell \gg 0, \ell \in \mathscr{S}$.

Proof. Because $\left|Z_{\ell}\right| \leq \gamma$ we see that (i) $\ell \not\left|Z_{\ell}\right|$ and (ii) $\ell \not\left|\operatorname{Aut}\left(Z_{\ell}\right)\right|$ for $\ell \gg 0, \ell \in \mathscr{S}$. From (i) and Schur-Zassenhauss, for any $\ell$-Sylow $S_{\ell} \subset G_{M_{\ell}}$, the group $Z_{\ell} S_{\ell}$ is a semidirect product $Z_{\ell} \rtimes S_{\ell}$ and, from (ii), the semidirect product $Z_{\ell} \rtimes S_{\ell}$ is actually a direct product that is $S_{\ell}$ is contained in the centralizer $Z_{G_{M_{\ell}}^{+}}\left(H_{\ell}\right)$ of $H_{\ell}$ in $G_{M_{\ell}}^{+}$. But, by definition, for $\ell \gg 0$ the group $G_{M_{\ell}}^{+}$is generated by the $\ell$-Sylow subgroups $S_{\ell}$ of $G_{M_{\ell}}$ hence $G_{M_{\ell}}^{+}=Z_{G_{M_{\ell}}^{+}}\left(Z_{\ell}\right)$.

Because $Z_{\ell}$ is commutative and of prime-to- $\ell$ order, $Z_{\ell}$ is conjugate in $\mathrm{GL}_{n}\left(\overline{\mathbb{F}}_{\ell}\right)$ to a diagonal torus. For any $z \in Z_{\ell}$ let $V P(z) \subset \overline{\mathbb{F}}_{\ell}^{\times}$denote the set of eigenvalues of $z$ and set

$$
V:=\prod_{z \in Z_{\ell}} V P(z)
$$


Then, $M \otimes_{\mathbb{F}_{\ell}} \overline{\mathbb{F}}_{\ell}$ can be decomposed into a direct sum

$$
M \otimes_{\mathbb{F}_{\ell}} \overline{\mathbb{F}}_{\ell}=\bigoplus_{\underline{\lambda} \in V} E(\underline{\lambda})
$$

where, for any $\underline{\lambda}=\left(\lambda_{z}\right)_{z \in Z_{\ell}}$ we write

$$
E(\underline{\lambda}):=\bigcap_{z \in Z_{\ell}} \operatorname{ker}\left(z-\lambda_{z} I d\right) .
$$

From the second claim, $G_{M_{\ell}}^{+}$stabilize each $\overline{\mathbb{F}}_{\ell}$-submodule $E(\underline{\lambda})$ of $M \otimes_{\mathbb{F}_{\ell}} \overline{\mathbb{F}}_{\ell}$ and from the first claim, it acts non diagonally at least on one of the nonzero $E(\underline{\lambda})$, say $E$. The action of $G_{M_{\ell}}^{+}$on $E$ induces a non trivial action on the projective space

$$
\mathbb{P}(E):=E / \overline{\mathbb{F}}_{\ell}^{\times}
$$

and, by definition of $E$, this action factors through $\bar{G}_{M_{\ell}}^{+}$. This shows that $\operatorname{PSL}_{2}\left(k_{r_{\ell}}\right)$ embeds into $\operatorname{PGL}(E)$. But $E$ is of $\overline{\mathbb{F}}_{\ell}$-dimension $\leq n$ so, from [LS74, Thm. p. 419], this can occur only for finitely many values of $r_{\ell}$. which, in turn, contradicts the fact that $r_{\ell} \rightarrow \infty$ for $\ell \in \mathscr{S}$ by Lemma 6 (2).

The proof of Theorem 1 is now complete.

\subsection{The case of $S_{\rho, 1}(\ell)$}

Whenever it is defined, we set for $i=1, \ldots, n=\operatorname{dim}_{\mathbb{F}_{\ell}}\left(H_{\ell}\right)$

$$
\gamma_{\rho, 1}^{i}(\ell):=\min \left\{\gamma_{v} ; 0 \neq v \in H_{\ell} \text { and } \operatorname{dim}_{\mathbb{F}_{\ell}}(M(v))=i\right\}
$$

Note that, when $n=i$, one has $\gamma_{\rho, 1}^{n}(\ell)=\gamma_{\rho, 1}(\ell)$.

Let $\mathscr{S}$ denote the set of all primes $\ell$ such that $H_{\ell}$ contains a $\pi_{1}(S)$-submodule of $\mathbb{F}_{\ell}$-rank 2 . Assume that $\mathscr{S}$ is infinite. In this section, we prove:

Proposition 14. Assume that conditions (WA), (I) and (T) are satisfied. Then:

$$
\lim _{\substack{\ell \rightarrow+\infty \\ p \ell\left(\ell^{2}-1\right)}} \gamma_{\rho, 1}^{2}(\ell)=+\infty .
$$

and deduce from this result the proof of Corollary 3. The proof of Proposition 14 needs some preparation.

We first study the possible structure of the group $G_{M}$ when $\operatorname{dim}_{\mathbb{F}_{\ell}}(M)=2$ and $\ell \gg 0$.

Lemma 15. Assume that conditions (WA), (I) and (T) are satisfied. Then, for $\ell \gg 0$ and any $\pi_{1}(S)$-submodule $M \subset H_{\ell}$ of $\mathbb{F}_{\ell}$-rank 2 one has $\operatorname{SL}(M) \subset G_{M}$. 
Proof. We write $G_{M}$ as an extension

$$
1 \rightarrow G_{M} \cap \mathrm{SL}(M) \rightarrow G_{M} \stackrel{\text { det }}{\longrightarrow} D_{M} \rightarrow 1,
$$

where $D_{M}=\operatorname{det}\left(G_{M}\right) \subset \mathbb{F}_{\ell}^{\times} \simeq \mathbb{Z} /(\ell-1)$.

Let us show first that $\left|G_{M} \cap \mathrm{SL}(M)\right|$ diverges with $\ell \rightarrow \infty$ in $\mathscr{S}$ and $M$ is any $\pi_{1}(S)$-submodule $M \subset H_{\ell}$ of $\mathbb{F}_{\ell}$-rank 2. Otherwise, up to replacing $\mathscr{S}$ by an infinite subset, we may assume that there exists an upper bound

$$
\left|G_{M} \cap \operatorname{SL}(M)\right| \leq B
$$

for all possible $M$. From Lemma 6 (2), one has

$$
\lim _{\substack{\ell \rightarrow+\infty \\ \ell \in \mathscr{S}}}\left|G_{M_{\ell}}\right|=+\infty
$$

which forces $\left|D_{M}\right|$ to diverge when $\ell \rightarrow \infty$ in $\mathscr{S}$. Let $O(B)$ denote the maximal order of the automorphism group of a group of order $\leq B$. Then, as $D_{M}$ is cyclic, it follows from Lemma 12 that $G_{M}$ contains a normal abelian subgroup of index $\leq B \cdot o(B)$, which contradicts Corollary 7 for $\ell \gg 0$ in $\mathscr{S}$.

Hence, for $\ell \gg 0$ in $\mathscr{S}$ and any $\pi_{1}(S)$-submodule $M \subset H_{\ell}$ of $\mathbb{F}_{\ell}$-rank 2, the only possibilities with respect to the list of Corollary 11 for $G_{M} \cap \operatorname{SL}(M)$ are (1), (2), (5) or (6). The types (1) and (2) are ruled out by condition (WA)' and Lemma 6 , and type (6) is exactly what the lemma claims. It remains to rule out type (5).

If $G_{M} \cap \mathrm{SL}(M)$ is of type (5), then it is contained in a Borel and thus fixes a line $\mathbb{F}_{\ell} \cdot v \subset M$ for some $0 \neq v \in H_{\ell}$. The line is uniquely determined since the $\ell$-Sylow of $G_{M} \cap \mathrm{SL}(M)$ is nontrivial, and thus $\mathbb{F}_{\ell} \cdot v$ is also invariant under $G_{M}$. However, by condition (WA) and Lemma 6 (2), the group $G_{M}$ cannot fix $\mathbb{F}_{\ell} \cdot v$, which is the desired contradiction.

Lemma 16. Assume that conditions (WA), (I) and (T) are satisfied. Then, there exists an integer $D \geq 1$ such that for $\ell \gg 0$ and any $\pi_{1}(S)$-submodule $M \subset H_{\ell}$ one has $\left|\operatorname{det}\left(G_{M}\right)\right| \leq D$.

Proof. Let $m$ denote the $\mathbb{F}_{\ell}$-rank of $M$. Then the action of $G_{M}$ on the line $\Lambda^{m} M$ factors through a faithfull action of $D_{M}:=\operatorname{det}\left(G_{M}\right)$. So the conclusion follows from condition (WA)'.

Now we can prove Proposition 14. Let $\mathscr{S}$ denote the set of all primes $\ell$ such that there exists $v \in H_{\ell}$ with $M(v)$ of $\mathbb{F}_{\ell}$-rank 2 . Assume that $\mathscr{S}$ is infinite and for every $\ell \in \mathscr{S}$, choose $v_{\ell} \in H_{\ell}$ with $M_{\ell}:=M\left(v_{\ell}\right)$ of $\mathbb{F}_{\ell}$-rank 2 such that $\gamma_{v_{\ell}}=\gamma_{\rho, 1}^{2}(\ell)$. By Lemma 15 and for $\ell \gg 0$ in $\mathscr{S}$ we write again $G_{M_{\ell}}$ as an extension

$$
1 \rightarrow \mathrm{SL}\left(M_{\ell}\right) \rightarrow G_{M_{\ell}} \stackrel{\text { det }}{\longrightarrow} D_{\ell} \rightarrow 1,
$$

where $D_{\ell}=\operatorname{det}\left(G_{M_{\ell}}\right) \subset \mathbb{F}_{\ell}^{\times} \simeq \mathbb{Z} /(\ell-1)$. From lemma 16, we have $\left|D_{\ell}\right| \leq D$. Consider an E-P decomposition 


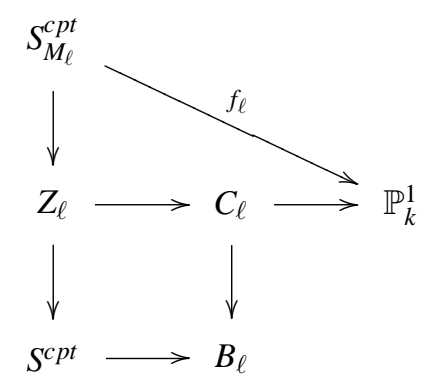

where $S_{M_{\ell}}^{c p t} \rightarrow Z_{\ell}=S_{M_{\ell}}^{c p t} / K_{\ell}$ and, with $\bar{G}_{M_{\ell}}=G_{M_{\ell}} / K_{\ell}$ faithfully acting on $C_{\ell}$, also $C_{\ell} \rightarrow B_{\ell}=C_{\ell} / \bar{G}_{M_{\ell}}$ are the respective quotient maps, and $\operatorname{deg}\left(f_{\ell}\right)=\gamma_{M_{\ell}}$. We set $D_{\ell}^{K}$ for the image of $K_{\ell}$ in $D_{\ell}$. Then $K_{\ell}$ fits into the short exact sequence

$$
1 \rightarrow K_{\ell} \cap \mathrm{SL}\left(M_{\ell}\right) \rightarrow K_{\ell} \rightarrow D_{\ell}^{K} \rightarrow 1
$$

As the only normal subgroups of $\mathrm{SL}_{2}\left(\mathbb{F}_{\ell}\right)$ are $1, \mathbb{Z} / 2$ and $\mathrm{SL}_{2}\left(\mathbb{F}_{\ell}\right)$, there are only two possibilities for $K_{\ell} \cap \operatorname{SL}\left(M_{\ell}\right)$, namely

(1) $K_{\ell} \cap \operatorname{SL}\left(M_{\ell}\right)=\operatorname{SL}\left(M_{\ell}\right)$.

(2) $K_{\ell} \cap \operatorname{SL}\left(M_{\ell}\right)=1, \mathbb{Z} / 2$,

In case (1), one has the estimate

$$
\gamma_{M_{\ell}}=\operatorname{deg}\left(f_{\ell}\right) \geq \operatorname{deg}\left(S_{M_{\ell}}^{c p t} \rightarrow Z_{\ell}\right)=\left|K_{\ell}\right|=\ell\left(\ell^{2}-1\right) \cdot\left|D_{\ell}^{K}\right|=\left|G_{M_{\ell}}\right| \cdot \frac{\left|D_{\ell}^{K}\right|}{\left|D_{\ell}\right|} .
$$

Since $\operatorname{SL}\left(M_{\ell}\right)$ acts transitively on $M_{\ell} \backslash\{0\}$, the stabilizer $G_{M_{\ell}, v_{\ell}}$ of $v_{\ell}$ under the action of $G_{M_{\ell}}$, namely the Galois group of $S_{M(v)} \rightarrow S_{v}$, has index $\ell^{2}-1$ and so

$$
\gamma_{\rho, 1}^{2}(\ell)=\gamma_{v_{\ell}} \geq \frac{\gamma_{M_{\ell}}}{\left|G_{M_{\ell}, v_{\ell}}\right|} \geq\left(\ell^{2}-1\right) \cdot \frac{\left|D_{\ell}^{K}\right|}{\left|D_{\ell}\right|} \geq \frac{\ell^{2}-1}{D} \rightarrow+\infty
$$

In case (2), the stabilizer has size

$$
\left|G_{M_{\ell}, v_{\ell}}\right|=\frac{\left|G_{M_{\ell}}\right|}{\ell^{2}-1}=\ell \cdot\left|D_{\ell}\right|
$$

and thus Lemma 8 applied to the primitive pair $\left(C_{\ell} \rightarrow B_{\ell}, C_{\ell} \rightarrow \mathbb{P}_{k}^{1}\right)$ in diagram (1.6) yields the estimate

$$
\gamma_{\rho, 1}^{2}(\ell)=\gamma_{v_{\ell}} \geq \frac{\gamma_{M_{\ell}}}{\left|G_{M_{\ell}, v_{\ell}}\right|} \geq \frac{\operatorname{deg}\left(S_{M_{\ell}}^{c p t} \rightarrow Z_{\ell}\right) \cdot \operatorname{deg}\left(C_{\ell} \rightarrow \mathbb{P}_{k}^{1}\right)}{\ell \cdot\left|D_{\ell}\right|} \geq \frac{\left|K_{\ell}\right| \cdot \sqrt{g_{C_{\ell}}+1}}{\ell \cdot\left|D_{\ell}\right|}
$$

For $\ell \gg 0$ and in particular $\ell>p$, the group $\bar{G}_{M_{\ell}}$ contains $\operatorname{SL}\left(M_{\ell}\right)$ or $\operatorname{PSL}\left(M_{\ell}\right)$, and so it is not a subgroup of the automorphism group of a curve of genus 0 or 1 over an algebraically closed field of characteristic $p \geq 0$. As a result, one may assume that 
$C_{\ell}$ has genus $\geq 2$. If $p$ does not divide $\ell\left(\ell^{2}-1\right)$ then $p$ does not divide $\left|\operatorname{GL}\left(M_{\ell}\right)\right|$ hence, a fortiori, does not divide $\left|\bar{G}_{M_{\ell}}\right|$. Consequently, the cover $C_{\ell} \rightarrow B_{\ell}$ lifts to characteristic 0 and we have the Hurwitz bound for the automorphism group

$$
\frac{\ell\left(\ell^{2}-1\right)\left|D_{\ell}\right|}{\left|K_{\ell}\right|}=\left|\bar{G}_{M_{\ell}}\right| \leq 84\left(g_{C_{\ell}}-1\right) .
$$

In combination with (1.7) this yields

$$
\gamma_{\rho, 1}^{2}(\ell) \geq \frac{\left|K_{\ell}\right| \cdot \sqrt{g_{C_{\ell}}+1}}{\ell \cdot\left|D_{\ell}\right|} \geq \frac{\left|K_{\ell}\right|}{\ell \cdot\left|D_{\ell}\right|} \sqrt{\frac{\ell\left(\ell^{2}-1\right)\left|D_{\ell}\right|}{84\left|K_{\ell}\right|}+2}
$$

Hence

$$
\gamma_{\rho, 1}^{2}(\ell) \geq \sqrt{\frac{\left(\ell^{2}-1\right)}{84 \cdot \ell \cdot|D|}} \rightarrow+\infty .
$$

This completes the proof of Proposition 14.

Remark 17. When $p \mid \ell\left(\ell^{2}-1\right)$, one can assert only that $\ell\left(\ell^{2}-1\right) \leq P_{p}\left(g_{C_{\ell}}\right)$ so the resulting lower bound for $g_{C_{\ell}}$ is too small to conclude. Also, from condition (T), one could observe that $Z_{\ell} \rightarrow S^{c p t}$ is tame for $\ell \gg 0$ but, if $p \mid \ell\left(\ell^{2}-1\right)$ and $S^{c p t} \rightarrow B_{\ell}$ is wildly ramified, it may happen that $C_{\ell} \rightarrow B_{\ell}$ is wildly ramified as well hence does not necessarily lift to characteristic 0 .

Finally, we give a proof of Corollary 3. Let $Y(0)$ and $Y_{1}(\ell)$ denote the coarse moduli schemes of the stack $\mathscr{E}$ of elliptic curves and of the stack $\mathscr{E}_{1}(\ell)$ of elliptic curves with a torsion point of order exactly $\ell$ as stacks over $k$. For any nonisotrivial relative elliptic curve $E \rightarrow S$ and $0 \neq v \in E_{\bar{\eta}}[\ell]$, one has the following commutative diagram

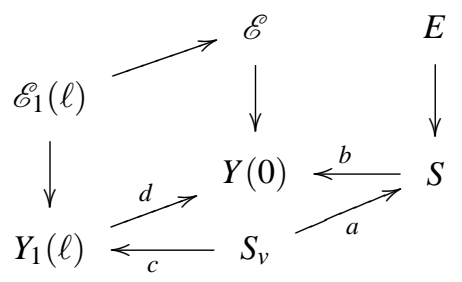

In particular we can estimate the gonality as

$$
\gamma_{Y_{1}(\ell)} \geq \frac{\gamma_{v}}{\operatorname{deg}(c)}=\frac{\gamma_{v} \operatorname{deg}(d)}{\operatorname{deg}(a) \operatorname{deg}(b)}=\frac{\gamma_{v}\left(\ell^{2}-1\right) / 2}{\left|G_{\ell} \cdot v\right| \operatorname{deg}(b)} \geq \frac{\gamma_{v}}{2 \operatorname{deg}(b)}
$$

with $\operatorname{deg}(b)$ independent of $v$ and $\ell$. Applying Proposition 14 to the family of rank-2 $\mathbb{F}_{\ell}$-linear representations

$$
\rho_{\ell}: \pi_{1}(S) \rightarrow \mathrm{GL}\left(E_{\bar{\eta}}[\ell]\right)
$$


gives the conclusion of Corollary 3 .

\section{References}

[A96] D. ABRAmovich, A linear lower bound on the gonality of modular curves, Internat. Math. Res. Notices 20, 1996, p. 1005-1011.

[CT10a] A. CADORET and A. TAMAGAWA, On a weak variant of the geometric torsion conjecture, preprint, 2010.

[CT10b] A. CADORET and A. TAMAGAWA, On a weak variant of the geometric torsion conjecture II, preprint, 2010.

[EHK10] J. ElLENBERG, C. HALL and E. KOWALSKI Expander graphs, gonality and variation of Galois representations, preprint, 2010.

[F91] G. FALtings, Diophantine approximation on abelian varieties, Annals of Math. 133, 1991, p. 549-576.

[Fr94] G. FREY Curves with infinitely many points of fixed degree, Israel J. Math. 85, 1994, p. 79-83.

[G83] O. GABbER Sur la torsion dans la cohomologie $\ell$-adique d'une variété, C.R. Acad. Sci. Paris Ser. I Math. 297, 1983, p. 179-182.

[LS74] V. LANDAZURI and G.M. SEITZ, On the minimal degree of projective representations of the finite Chevalley groups, J. Algebra 32, 1974, p. 418-443.

[N87] M.V. NORI, On subgroups of $\mathrm{GL}_{n}\left(\mathbb{F}_{p}\right)$, Inventiones. Math. 88, p. 257-275, 1987.

[P07] B. PoOnEn, Gonality of modular curves in characteristic p, Math. Res. Letters 14, 2007, p. 691-701.

[St73] H. Stichtenoth, Über die Automorphismengruppe eines algebraischen Funktionenkörpers von Primzahlcharakteristik I, II, Arch. der Math. (Basel) 24, 1973, p. 524-544 and p. 615-631.

[Su82] M. SuZUKI, Group theory I, Grundlehren der Mathematischen Wissenschaften 247, Springer-Verlag, 1982.

[T04] A. TAMAGAWA, Finiteness of isomorphism classes of curves in positive characteristic with prescribed fundamental group, J. Algebraic Geometry 13, 2004, p. 675-724.

anna.cadoret@math.u-bordeauxl.fr

Institut de Mathématiques de Bordeaux - Université Bordeaux 1,

351 Cours de la Libération,

F33405 TALENCE cedex, FRANCE. 